\title{
Interaction of Warfarin with Human Serum Albumin and Effect of Ferulic Acid on the Binding
}

\author{
Qiang Li, ${ }^{1,2}$ Wen-yue Yang, ${ }^{3}$ Ling-ling Qu, ${ }^{3}$ Huan-Yang Qi, ${ }^{4}$ \\ Yun Huang, ${ }^{3}$ and Zheng Zhang ${ }^{1,2}$ \\ ${ }^{1}$ Heart Center, The First Hospital of Lanzhou University, Lanzhou, Gansu 730000, China \\ ${ }^{2}$ The First Clinical Medical College of Lanzhou University, Lanzhou, Gansu 730000, China \\ ${ }^{3}$ Pharmaceutical College, Hebei Medical University, Shijiazhuang 050017, China \\ ${ }^{4}$ Lanzhou Institute of Chemical Physics, Chinese Academy of Sciences, Lanzhou, Gansu 730000, China
}

Correspondence should be addressed to Yun Huang; hy9317536@126.com and Zheng Zhang; zhangccu@163.com

Received 20 December 2013; Accepted 3 February 2014; Published 13 March 2014

Academic Editor: Lu Yang

Copyright (C) 2014 Qiang Li et al. This is an open access article distributed under the Creative Commons Attribution License, which permits unrestricted use, distribution, and reproduction in any medium, provided the original work is properly cited.

\begin{abstract}
Angelica sinensis (Oliv.) Diels combined treatment with warfarin would increase the risk of bleeding. Ferulic acid is an abundant hydroxycinnamic acid in A. sinensis and warfarin is the most widely used oral anticoagulant. The studies on supermolecular interaction of warfarin with human serum albumin (HSA) and the influence of ferulic acid on the binding would contribute to the understanding of the metabolic processes of warfarin and the effect of ferulic acid. We focus on investigating the effect of warfarin on fluorescence spectrum of human serum albumin (HSA), fluorescence quenching mechanism, binding constant, Hill coefficient, binding mode, and the effect of different ferulic acid concentrations on the binding. Warfarin quenched the intrinsic fluorescence of HSA mainly by static quenching. Accession of ferulic acid reduced the binding of HSA-warfarin. By decreasing binding constant and the Hill coefficient of warfarin with HSA, ferulic acid could improve the plasma concentration of free warfarin, which would increase the risk of bleeding. Warfarin's free concentration increased at least $50 \%$ under the condition of simulated human body. The results indicated that $A$. sinensis combined treatment with warfarin would increase the risk of bleeding. And the results provide an important theoretical support for warfarin used as oral anticoagulant.
\end{abstract}

\section{Introduction}

Angelica sinensis (Oliv.) Diels, which belongs to the genus Angelica (family Apiaceae), was first documented in Shennong Bencao Jing (edited in Han Dynasty in China) and its root is known in Chinese as Danggui or Dongquai. A. sinensis is a popular traditional Chinese medicinal (TCM) herb which is easily obtained by the public [1-3]. Its roots as a Chinese herbal supplement are used for treatment of menstrual cramping, irregular menses, and menopausal symptoms. A. sinensis is used alone, or in combination with other herbs, as an antispasmodic, a "blood purifier," and a tonic to strengthen the heart, lung, and liver meridians. A. sinensis is also used as a health food product in Asia and as a dietary supplement in Europe and America for the treatment of a variety of gynecological problems [4].
Ferulic acid (Figure 1), the phenolic acid, which is believed to be one of the most biologically active components in A. sinensis, is used as a marker compound to evaluate the quality of $A$. sinensis in Chinese Pharmacopoeia [5-7]. In vivo and in vitro studies showed that ferulic acid from $A$. sinensis had antithrombotic activity. Ferulic acid interrupts platelet polymerization by inhibiting the release of serotonin and adenosine diphosphate from platelets and by reducing production of thromboxane A2 (TXA2) through inhibiting cyclooxygenase and thromboxane synthetase in arachidonic acid metabolism [8].

Warfarin (Figure 1), the 4-hydroxycoumarin compound, is the most widely used oral anticoagulant. Onset and duration of the action are rapid and predictable, and its bioavailability is excellent. Warfarin is highly water soluble and rapidly absorbed from the stomach and the upper 
<smiles>CC(=O)CC(c1ccccc1)c1c(O)c2ccccc2oc1=O</smiles>

Warfarin<smiles>COc1cc(/C=C/C(=O)O)ccc1O</smiles>

Ferulic acid

FIGURE 1: The tentative structure of warfarin and ferulic acid.

gastrointestinal tract. Its plasma concentrations peak occurs 60 to 90 minutes after oral administration. Warfarin binding to the enzyme vitamin $\mathrm{K}$ 2,3-epoxide reductase in liver microsomes stops the cycle of vitamin $\mathrm{K}$ and reduces gammacarboxylation of the precursors of vitamin D-dependent proand anticoagulant factors [9].

In 2000, a review "Herb-drug interactions" in Lancet indicated that Chinese traditional herb A. sinensis combined treatment with warfarin would increase the risk of bleeding [10]. In 2002 and 2010, there were two reviews in JACC commented the drug interactions between $A$. sinensis and warfarin. Case reports were also suggestive of interaction between warfarin and A. sinensis [11, 12]. A patient stabilized on warfarin experienced an increase in prothrombin time (PT) and international normalized ratio (INR) after taking $A$. sinensis for perimenopausal symptoms [8].

For the clinical studies, several cases published previously showed gross over-anticoagulation and bleeding complications on patients received chronic warfarin therapy in combination with $A$. sinensis. However, until now, the mechanism of interactions of $A$. sinensis and warfarin was not clear and little attention has been paid to the effect of ferulic acid on warfarin binding with human serum albumin (HSA). HSA is the most abundant protein constituent of blood plasma and serves as a protein storage component. As one of the most extensively studied model proteins, HSA has high affinity to many endogenous and exogenous compounds, serving in transporting drugs or other organic molecules to their targets $[13,14]$. Therefore, investigating the interaction of active components in Chinese herbs with HSA can provide useful information of drug actions and can be used as a model for elucidating the drug-protein complex [15].

In order to further explore whether the interactions between warfarin and HSA occur and the influence of ferulic acid on them, we primarily focus on investigating the effect of warfarin on fluorescence spectrum of HSA, fluorescence quenching mechanism, binding constant, Hill coefficient, binding mode, and the effect of different ferulic acid concentrations on the binding, which are expected to provide more information about the interaction between warfarin and HSA. The studies on supermolecular interaction of warfarin with HSA and the influence of ferulic acid on the binding would contribute to the understanding of the metabolic processes of warfarin. And the results may provide an important theoretical support for warfarin used as oral anticoagulant.

\section{Materials and Methods}

2.1. Materials. HSA purchased from Sigma (New York, USA) was $98.0-99.0 \%$ pure (agarose gel electrophoresis) and Tris (no less than $99.5 \%$ pure) was purchased from Sigma. Ferulic acid and warfarin (no less than $98.0 \%$ pure) were bought from the National Institute for the Control of Pharmaceutical and Biological Products (Beijing, China). All other chemicals were from Beijing Chemical Reagents Company and were of analytical reagent grade. Double distilled water was used throughout the experiment.

2.2. Apparatus. Fluorescence measurements were performed on a Perkin-Elmer spectrofluorimeter Model LS-55 equipped with a $150 \mathrm{~W}$ Xenon lamp and a thermostat bath. The widths of both the excitation slit and emission slit were set at $5.0 \mathrm{~nm}$. The absorption spectra were measured on a Shimadzu UV$2450 \mathrm{UV}$-Vis spectrophotometer. The $\mathrm{pH}$ measurements were carried out on a PHS-3C digital pH meter (Shanghai Leici Instrument Factory). Titrations were done manually by using trace syringes. A quartz cell of $1.00 \mathrm{~cm}$ pathlength was used for the measurements.

2.3. Procedures. HSA $\left(5 \times 10^{-4} \mathrm{M}\right)$, warfarin $\left(1 \times 10^{-3} \mathrm{M}\right)$, and ferulic acid $\left(1 \times 10^{-3} \mathrm{M}\right)$ were diluted with Tris- $\mathrm{HCl}$ buffer $(0.05 \mathrm{M}, \mathrm{pH} 7.40$, containing $0.10 \mathrm{M} \mathrm{NaCl})$ to some lower concentration for actual use. A $5 \times 10^{-4} \mathrm{M}$ HSA stock solution was diluted to some lower concentrations with Tris$\mathrm{HCl}$ buffer solution. The final concentrations of HSA varied from 0 to $5 \times 10^{-6} \mathrm{M} .3 \mathrm{~mL}$ of HSA $\left(4 \times 10^{-6} \mathrm{M}\right)$ was titrated to give $0,6,12,18,24,30,36,42$, or $48 \mu \mathrm{L}$ of warfarin $(1 \times$ $\left.10^{-3} \mathrm{M}\right)$, respectively, and a certain amount of Tris- $\mathrm{HCl}$ buffer solution to give the final volume of $3.05 \mathrm{~mL}$. Another solution containing HSA in the absence and presence of ferulic acid $\left(6 \times 10^{-6} \mathrm{M}\right)$ was prepared similarly. The final concentrations of warfarin varied from 0 to $16 \times 10^{-6} \mathrm{M}$ at an increment of $2 \times 10^{-6} \mathrm{M}$. The resultant mixture was subsequently ultrasonicated for $1 \mathrm{~min}$ and incubated for $5 \mathrm{~min}$ at 298 or $310 \mathrm{~K}$.

Fluorescence spectra were recorded at 298 and $310 \mathrm{~K}$ in the range from 300 to $450 \mathrm{~nm}$ at excitation wavelength $287 \mathrm{~nm}$. The synchronous fluorescence spectra were recorded when $\Delta \lambda=15 \mathrm{~nm}, \Delta \lambda=60 \mathrm{~nm}$, and $\Delta \lambda=90 \mathrm{~nm}$. The threedimensional fluorescence spectra were performed under the following conditions: emission wavelengths over a range of 280-450 nm, excitation wavelengths over a range of 220$320 \mathrm{~nm}$ with an increment of $2 \mathrm{~nm}$. The UV-Vis absorption 


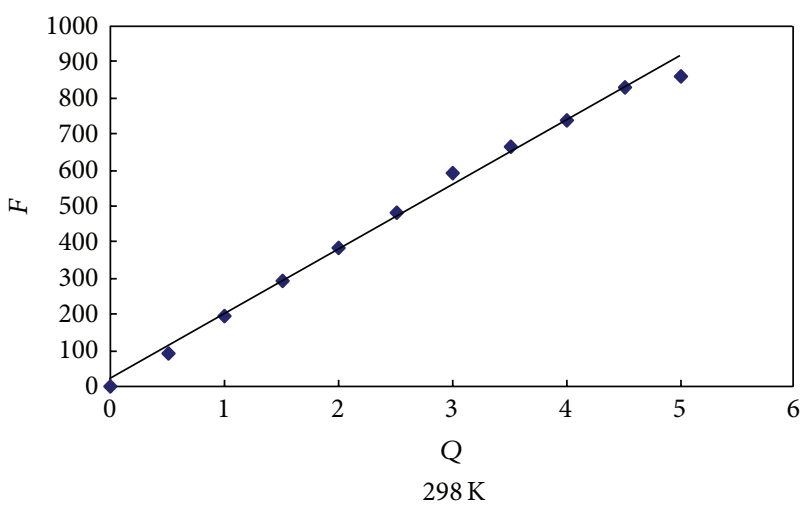

(a)

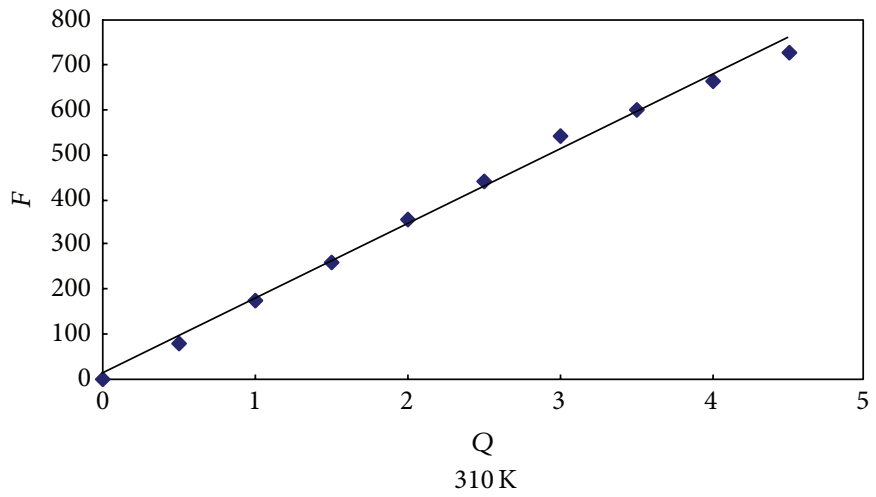

(b)

FIgURE 2: Standard curve of HSA at 298 and $310 \mathrm{~K}$ when $\Delta \lambda=60 \mathrm{~nm}, C_{(\mathrm{HSA})}=0,0.5,1,1.5,2,2.5,3,3.5,4,4.5,5 \times 10^{-6} \mathrm{M}$.

spectrum of warfarin (HSA or warfarin-ferulic acid) was recorded in the range from 280 to $450 \mathrm{~nm}$ using UV-2450.

\section{Results and Discussion}

3.1. Determination of Free HSA Concentration. Synchronous fluorescence spectrum shows the fluorescence of Trp residues when $\Delta \lambda=60 \mathrm{~nm}$. The intrinsic fluorescence is mainly manifested by emission of Trp residues in molecule [16]. In this research, the standard curve of HSA was got from synchronous fluorescence spectrum of Trp residues. So the free concentration of HSA can be derived from the standard curve of HSA. The standard curves of HSA were shown in Figure 2 and Table 1.

\subsection{Interaction of HSA with Warfarin}

3.2.1. Effect of Warfarin on Fluorescence Spectrum of HSA. Figure 3 shows the fluorescence quenching spectra of HSA with different concentrations of warfarin, suggesting that warfarin could combine with HSA. The fluorescence intensity of HSA at $344 \mathrm{~nm}$ decreased regularly with the increase of warfarin concentration, suggesting that the complex of warfarin-HSA was formed, which is responsible for quenching HSA fluorescence.

3.2.2. Binding Rate of Warfarin with HSA. According to the literature, the proportion of HSA, warfarin, and ferulic acid in human body is $100: 0.5: 1$. And $99.9 \%$ of warfarin combines with HSA in blood $[17,18]$. So the concentrations of warfarin and ferulic acid are $2 \times 10^{-8} \mathrm{M}$ and $3 \times 10^{-8} \mathrm{M}$, respectively, when the concentration of HSA is $4 \times 10^{-6} \mathrm{M}$. The binding rate of HSA with warfarin is very high. And the amount of HSA is much more than warfarin in this research. So it can be concluded that almost all of warfarin was combined with HSA.

Figure 4 shows that synchronous fluorescence intensity was declined after warfarin was added. The fluorescence intensity was 759 and 754 when warfarin was absent and present. The free concentration of HSA was $3.98 \times 10^{-6} \mathrm{M}$
TABLE 1: Equation of standard curve of HSA when $\Delta \lambda=60 \mathrm{~nm}$.

\begin{tabular}{lcc}
\hline$T / \mathrm{K}$ & Equation & $R^{2}$ \\
\hline $298 \mathrm{~K}$ & $F=178.44[Q]+20.636$ & 0.9941 \\
$310 \mathrm{~K}$ & $F=165.35[Q]+11.473$ & 0.9953 \\
\hline
\end{tabular}

when warfarin was added according to the equations of Table 1 . The free concentration of HSA was reduced by $2 \times$ $10^{-8} \mathrm{M}$ and the concentration of warfarin was $2 \times 10^{-8} \mathrm{M}$. So it can be speculated that the binding site of warfarin with HSA was $1: 1$. And then ferulic acid was added and the fluorescence intensity was $755 \mathrm{~nm}$. At this point, the free concentration of HSA was $3.99 \times 10^{-6} \mathrm{M}$ according to the equations of Table 1 . It did not only decrease, but also increased to $1 \times 10^{-8} \mathrm{M}$. So it can be speculated that the accession of ferulic acid reduced the binding of HSA-warfarin. Under the condition of simulated human body, the free concentration of warfarin increased at least $50 \%$.

\subsubsection{Fluorescence Quenching Mechanism and Quenching} Constant. The quenching mechanism can be interpreted by the fluorescence quenching spectra of the protein and the Stern-Volmer plots of warfarin with HSA as shown in Figure 3. The procedure of the fluorescence quenching is assumed to be dynamic quenching in order to confirm the view. The Stern-Volmer equation is described as

$$
\frac{F_{0}}{F}=1+K_{q} \tau_{0}[Q]=1+K_{\mathrm{SV}}[Q],
$$

where $F_{0}$ and $F$ are the relative fluorescence intensities in the absence and presence of quencher, $K_{q}$ is the quenching rate constant of biomolecule, $\tau_{0}$ is the average lifetime of the biomolecule $\left(10^{-8} \mathrm{~s}\right)$ without quencher, and $[Q]$ is the free concentration of the quencher. $K_{\mathrm{SV}}$ is the dynamic quenching constant [19]. Dynamic and static quenching can be distinguished by their different dependence on temperature. The $K_{\mathrm{SV}}$ values decrease with an increase in temperature for static quenching, and the reverse effect would be observed for dynamic quenching [20]. The Stern-Volmer plots from our 


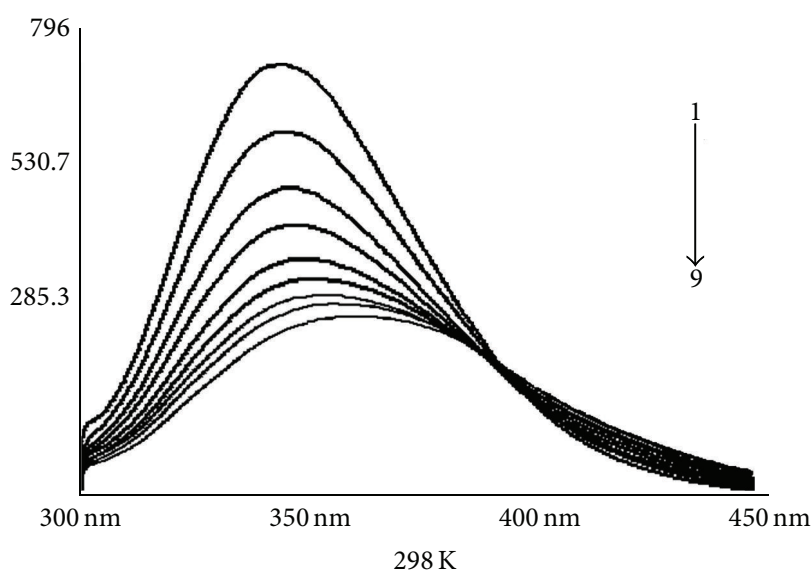

(a)

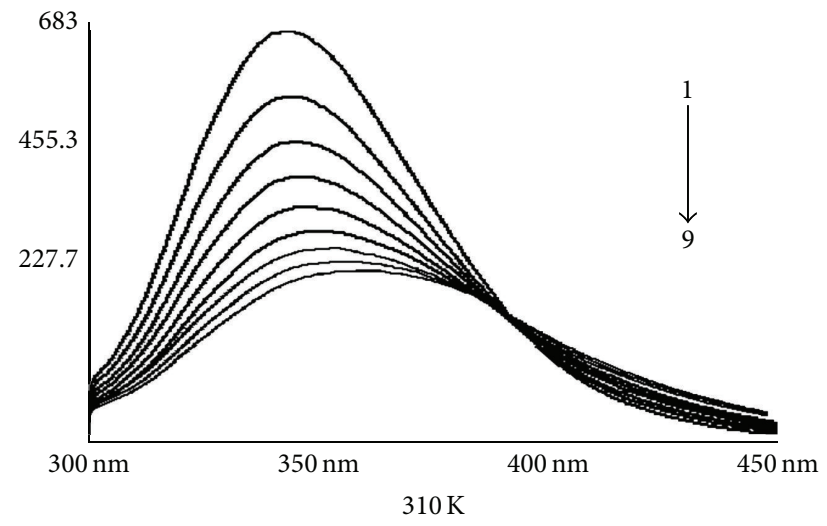

(b)

FIGURE 3: Effect of warfarin on fluorescence spectrum of HSA; form curve $1 \rightarrow 9, C_{(\mathrm{HSA})}=4 \times 10^{-6} \mathrm{M}$, and $C_{(\text {warfarin) }}=$ $0,2,4,6,8,10,12,14,16 \times 10^{-6} \mathrm{M}$, respectively.

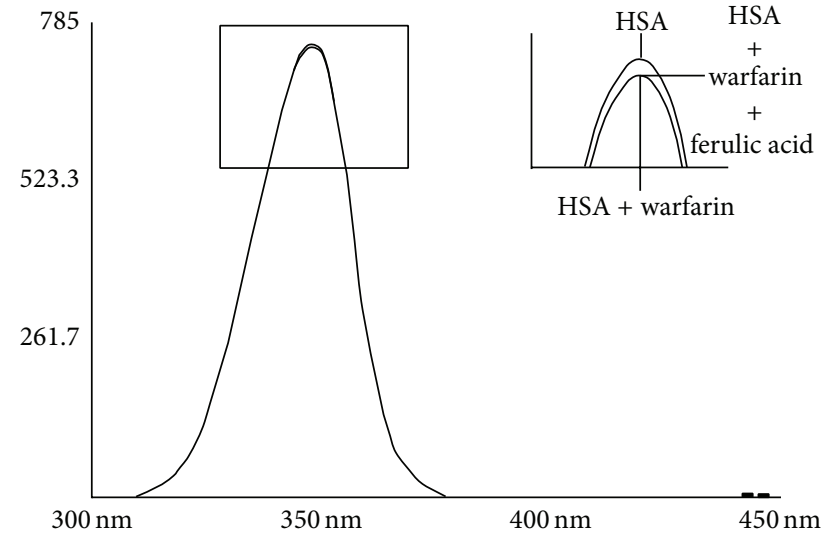

FIGURE 4: Synchronous fluorescence of effect of absence and presence of ferulic acid on HSA-warfarin, $C_{(\mathrm{HSA})}=4 \times 10^{-6} \mathrm{M}$, $C_{(\text {warfarin })}=2 \times 10^{-8} \mathrm{M}$, and $C_{(\text {ferulic acid) }}=3 \times 10^{-8} \mathrm{M}$.

data (Table 2 and Figure 5) had good linearity and the slope declined $\left(K_{\mathrm{SV}}\right.$ from $1.22 \times 10^{5}$ to $\left.1.18 \times 10^{5}\right)$ with increasing temperature. The result suggests that warfarin effectively quenched the intrinsic fluorescence of HSA mainly by static quenching. The static quenching constant $K_{\mathrm{LB}}$ (Table 2 ) was developed by the Lineweaver-Burk equation

$$
\left(F_{0}-F\right)^{-1}=F_{0}^{-1}+K_{\mathrm{LB}}^{-1} F_{0}^{-1}[Q]^{-1} .
$$

The UV-Vis absorption spectra of HSA with warfarin were shown in Figure 6 . We found that the maximum absorption wavelengths of HSA were located at $278 \mathrm{~nm}$ when warfarin was absent and present. The UV absorbance intensity of HSA was increased with the addition of warfarin, which indicates the formation of a complex between HSA with warfarin and change in protein conformation [21]. This result once again confirmed that warfarin effectively quenched the intrinsic fluorescence of HSA mainly by static quenching.

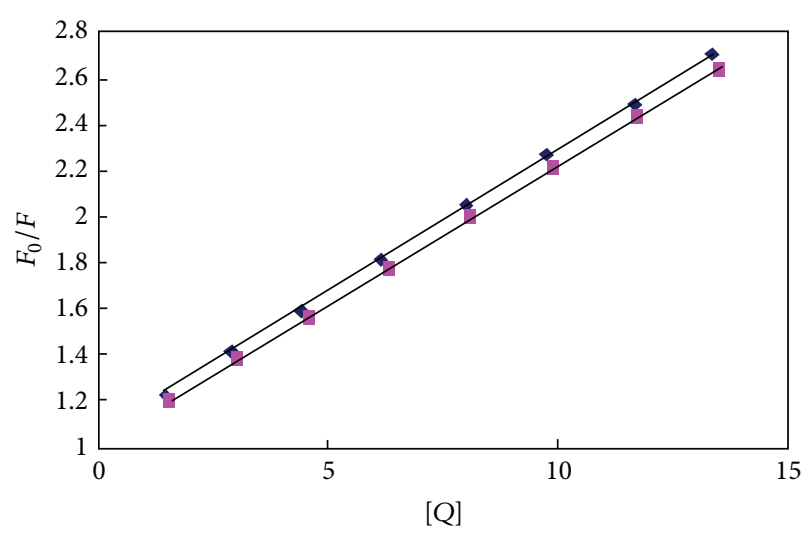

- $298 \mathrm{~K}$

- $310 \mathrm{~K}$

FIGURE 5: Stern-Volmer plots of warfarin-HSA at different temperatures.

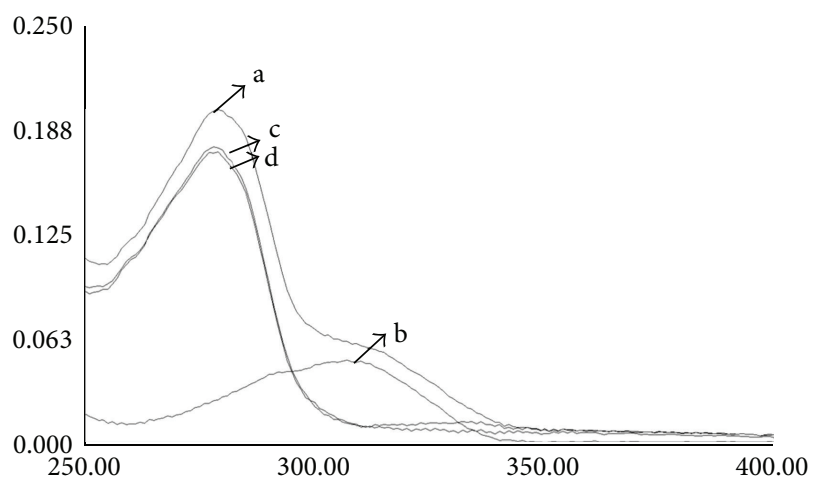

FIGURE 6: The UV absorption spectra of HSA-warfarin (a), warfarin (b), (HSA-warfarin)-warfarin (c), and HSA (d) $C_{(\text {HSA })}=C_{(\text {warfarin })}=$ $4 \times 10^{-6} \mathrm{M}$. 
TABLE 2: The binding parameters of warfarin-HSA at different temperatures.

\begin{tabular}{lcccr}
\hline \multirow{2}{*}{$T / \mathrm{K}$} & Stern-Volmer & Lineweaver-Burk & \multicolumn{2}{c}{ Double logarithm } \\
& $K_{\mathrm{sv}}(\mathrm{M})$ & $K_{\mathrm{LB}}$ & $8.42 \times 10^{4}$ & $n_{H}$ \\
\hline $298 \mathrm{~K}$ & $1.22 \times 10^{5}$ & $1.30 \times 10^{5}$ & 6.9643 & 0.9969 \\
$310 \mathrm{~K}$ & $1.18 \times 10^{5}$ & $1.39 \times 10^{5}$ & $0.17 \times 10^{4}$ & 0.9428 \\
\hline
\end{tabular}

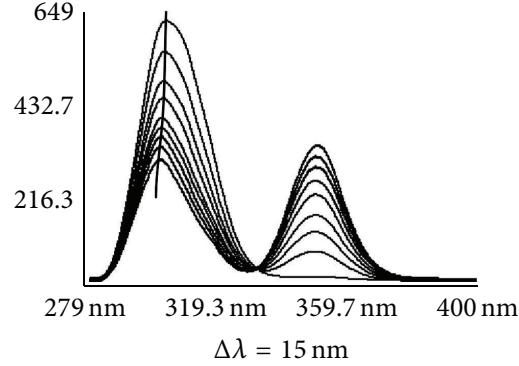

(a)

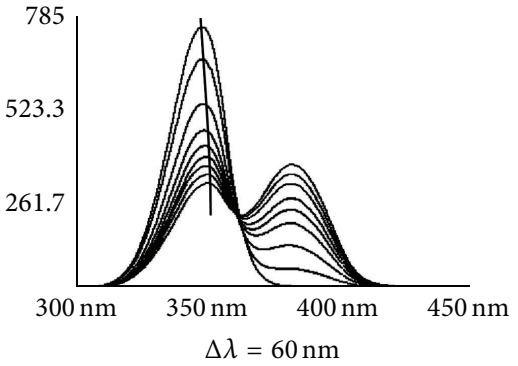

(b)

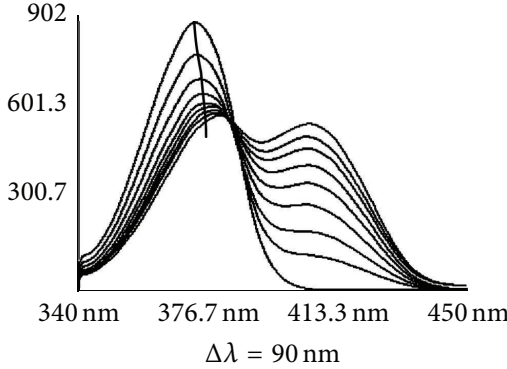

(c)

FIGURE 7: Synchronous fluorescence spectra of interaction between HSA and warfarin.

3.2.4. The Binding Constant, Hill Coefficient, and Binding Distance. For the static quenching interaction, if it is assumed that there are similar and independent binding sites in the biomacromolecule, binding constant $\left(K_{a}\right)$, and the Hill coefficient $\left(n_{H}\right)$ can be got from the double logarithm regression curve equation

$$
\lg \frac{\left(F_{0}-F\right)}{F}=\lg K_{a}+n_{H} \lg [Q] .
$$

Table 2 gives the values of $K_{a}$ and $n_{H}$ at 298 and $310 \mathrm{~K}$.

3.2.5. Conformation Investigation. Synchronous fluorescence spectrum can simplify the spectrum, narrow band, and reduce the spectral overlap. It shows the fluorescence of Tyr residues when $\Delta \lambda=15 \mathrm{~nm}, \operatorname{Trp}$ residues when $\Delta \lambda=$ $60 \mathrm{~nm}$, and Phe residues when $\Delta \lambda=90 \mathrm{~nm}$. The change of the position of synchronous fluorescence reflects the change of surrounding microenvironment polarity of amino acid residues [22-24]. If the $\lambda_{\mathrm{em}}$ exhibits blueshift, it indicates that the microenvironment polarity of amino acid residues was decreased and hydrophobicity was increased. And redshift, on the other hand, indicates that the microenvironment polarity of amino acid residues was increased and hydrophobicity was decreased. Three synchronous fluorescence spetra were shown in Figure 7. It can be learned that the maximum emission wavelength of Trp and Phe residues had a significant redshift and Tyr residues had a buleshift. It indicated that the conformation of HSA was changed, the polarity around the Trp and Phe residues was increased, and the hydrophobicity was decreased. And the Tyr residues, on the other hand, were decreased, and the hydrophobicity was increased.

3.2.6. Binding Forces. The interaction forces between small organic molecular and biomacromolecule mainly involve hydrogen bonds, Vander Waals forces, electrostatic interactions, hydrophobic forces, and so forth. According to the
TABLE 3: Thermodynamic parameter of interaction between HSA and warfarin.

\begin{tabular}{lccc}
\hline$T / \mathrm{K}$ & $\Delta H\left(\mathrm{KJ} \cdot \mathrm{mol}^{-1}\right)$ & $\Delta S\left(\mathrm{~J} \cdot \mathrm{k}^{-1}\right)$ & $\Delta G\left(\mathrm{KJ} \cdot \mathrm{mol}^{-1}\right)$ \\
\hline 298 & \multirow{2}{*}{4.28} & 112.26 & -29.17 \\
310 & & 112.26 & -30.52 \\
\hline
\end{tabular}

views of Ross and Subramanian, $\Delta H>0$ and $\Delta S>0$ imply a hydrophobic interaction, $\Delta H<0$ and $\Delta S<0$ reflect hydrogen bonding and van der Waals interaction, and $\Delta H<0$ and $\Delta S>0$ imply electrostatic force. Consider thermodynamic equations [25]

$$
\begin{aligned}
\ln \left(\frac{K_{\mathrm{LB} 2}}{K_{\mathrm{LB} 1}}\right) & =\left(\frac{1}{T 1}-\frac{1}{T 2}\right) \frac{\Delta H}{R}, \\
\Delta G & =\Delta H-T \Delta S, \\
\Delta G & =-R T \ln K_{\mathrm{LB}} .
\end{aligned}
$$

The values of $\Delta H, \Delta S$, and free energy change $(\Delta G)$ for warfarin binding to HSA are listed in Table 3. Consequently, the positive $\Delta H$ and positive $\Delta S$ values suggested that hydrophobic interaction played major roles in warfarin binding to HSA. The negative value of $\Delta G$ reveals that the interaction process was spontaneous.

3.3. Effect of Ferulic Acid on Binding Constant and Hill Coefficient. Figure 8 shows the fluorescence spectra of warfarinHSA in the presence of ferulic acid. According to (3), the $K_{a}$ and $n_{H}$ can be got as $K_{a}=6.53 \times 10^{4}$ and $n=0.9505$. It can be learned that the $K_{a}$ and $n_{H}$ were decreased after ferulic acid was added.

Figure 9 presents the contour spectra of (a) HSA, (b) HSA-warfarin, and (c) HSA-warfarin-ferulic acid, respectively. Peak 1 is the Rayleigh scattering peak $\left(\lambda_{\text {ex }}=\lambda_{\text {em }}\right)$, and peak 2 is the spectral behavior of Trp residues. The stoke shifts 
TABLE 4: Contour spectra characteristics of HSA, HSA-warfarin, and HSA-warfarin-ferulic acid systems.

\begin{tabular}{lccc}
\hline System & Peak 2 $\left(\lambda_{\mathrm{ex}} / \lambda_{\mathrm{em}}\right)$ & $\Delta \lambda(\mathrm{nm})$ & \\
\hline HSA & $284 / 336$ & 52 & Intensity \\
HSA-warfarin & $284 / 338$ & 54 & 362 \\
HSA-warfarin-ferulic acid & $284 / 339$ & 55 & 397 \\
\hline
\end{tabular}

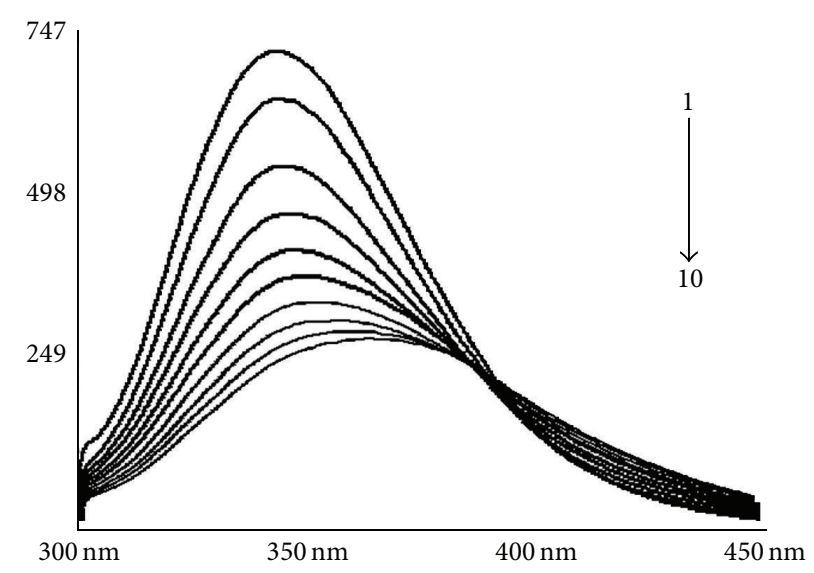

FIGURE 8: Fluorescence spectra of the effect of ferulic acid on warfarin-HSA, form curve $1 \rightarrow 10, C_{(\mathrm{HSA})}=4 \times 10^{-6} \mathrm{M}, C_{(\mathrm{ferulic} \mathrm{acid})}=6 \times 10^{-6} \mathrm{M}$, and $C_{(\text {warfarin) }}=0,2,4,6,8,10,12,14,16 \times 10^{-6} \mathrm{M}$, respectively.

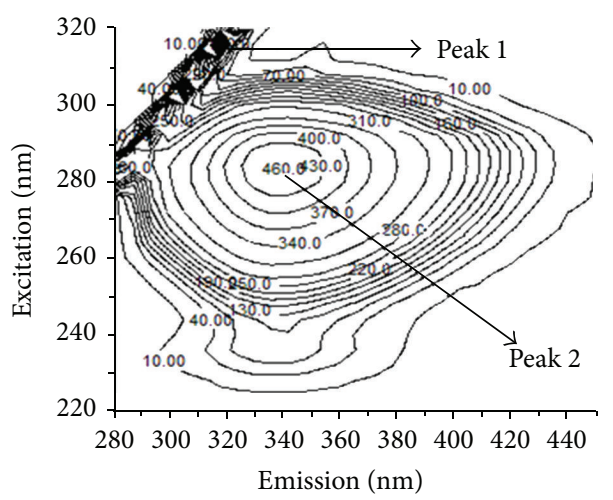

(a)

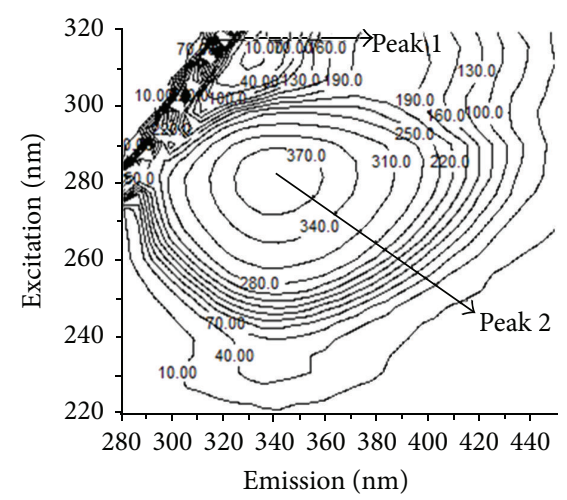

(b)

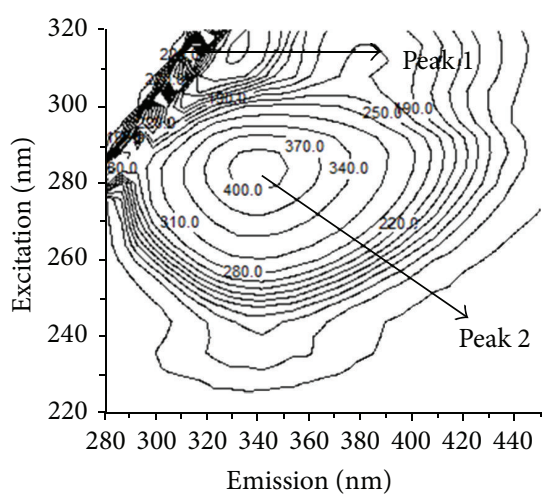

(c)

FIGURE 9: Contour spectra of (a) HSA, (b) HSA-warfarin, and (c) HSA-warfarin-ferulic acid. $C_{(\mathrm{HSA})}=C_{(\text {warfarin })}=4 \times 10^{-6} \mathrm{M}, C_{(\text {ferulic acid) }}=$ $6 \times 10^{-6} \mathrm{M}$.

$(\Delta \lambda)$ and density of contour have been changed after warfarin and ferulic acid were added (Table 4 ), which indicated that the interactions between them induced microenvironment and conformation changes in HSA. The fluorescence intensities of peak 2 decreased obviously in the presence of warfarin or ferulic acid. Redshift in the presence of warfarin or ferulic acid revealed the Trp residues were brought to a more hydrophilic environment [16].

\section{Conclusions}

Angelica sinensis (Oliv.) Diels is a popular traditional Chinese medicine (TCM) which is easily obtained by the public not only for medicinal use but also for a dietary supplement. Ferulic acid, [3-(4-hydroxy-3-methoxyphenyl)-2-propenoic acid], is an abundant hydroxycinnamic acid and one of the most biologically active components in A. sinensis [26]. Warfarin, 4-hydroxy-3-(3-oxo-1-phenylbutyl)-2H-1-benzopyran2 -one, is the most widely used oral anticoagulant. This paper presents the interaction of warfarin with HSA and effect of ferulic acid on the binding by fluorescence emission and synchronous fluorescence. By decreasing binding constant and the Hill coefficient of warfarin with HSA, ferulic acid could improve the plasma concentration of free warfarin, which would increase the risk of bleeding. Under the condition of simulated human body, the free concentration of 
warfarin increased at least $50 \%$. It is very dangerous for the patient stabilized on warfarin because of its narrow therapeutic window. The studies on supermolecular interaction of warfarin with HSA and the influence of ferulic acid on the binding would contribute to the understanding of the metabolic processes of warfarin and the effect of ferulic acid. The results indicated that $A$. sinensis combined treatment with warfarin would increase the risk of bleeding. And the results provide an important theoretical support for warfarin used as oral anticoagulant.

\section{Conflict of Interests}

The authors declare that there is no conflict of interests regarding the publication of this paper.

\section{Acknowledgments}

The authors gratefully acknowledge financial support of the Natural Science Foundation of Hebei Province (no. 08B033), the Natural Science Foundation of Gansu Province (no. 1010RJZA123), and the Fundamental Research Funds for the Central Universities (lzujbky-2011-102).

\section{References}

[1] K. J. Zhao, T. T. X. Dong, P. F. Tu, Z. H. Song, C. K. Lo, and K. W. K. Tsim, "Molecular genetic and chemical assessment of radix Angelica (Danggui) in China," Journal of Agricultural and Food Chemistry, vol. 51, no. 9, pp. 2576-2583, 2003.

[2] X. B. Yang, Y. Zhao, and Y. Lv, "In vivo macrophage activation and physicochemical property of the different polysaccharide fractions purified from Angelica sinensis," Carbohydrate Polymers, vol. 71, no. 3, pp. 372-379, 2008.

[3] S. X. Deng, S.-N. Chen, P. Yao et al., "Serotonergic activityguided phytochemical investigation of the roots of Angelica sinensis," Journal of Natural Products, vol. 69, no. 4, pp. 536-541, 2006.

[4] Q. Fan, P. F. Xia, X. Liu, J. H. Gu, X. Y. Wu, and L. Zhao, "Simultaneous quantification of two major active components in Radix Angelica sinensis by HPLC with an internal standard correction method," Instrumentation Science \& Technology, vol. 40, no. 5, pp. 416-428, 2012.

[5] Y.-L. Wang, Y.-Z. Liang, B.-M. Chen, Y.-K. He, B.-Y. Li, and Q.-N. Hu, "LC-DAD-APCI-MS-based screening and analysis of the absorption and metabolite components in plasma from a rabbit administered an oral solution of danggui," Analytical and Bioanalytical Chemistry, vol. 383, no. 2, pp. 247-254, 2005.

[6] H. Wang, L. Kong, H. F. Zou, J. Y. Ni, and Y. K. Zhang, "Screening and analysis of biologically active compounds in Angelica sinensis by molecular biochromatography," Chromatographia, vol. 50, no. 7-8, pp. 439-445, 1999.

[7] L. M. Shao, Pharmacopoeia of the People's Republic of China (Part I), Chemical Industry Press, Beijing, China, 2010.

[8] R. L. Page II and J. D. Lawrence, "Potentiation of warfarin by Dong Quai,” Pharmacotherapy, vol. 19, no. 7, pp. 870-876, 1999.

[9] G. Palareti and C. Legnani, "Warfarin withdrawal: pharmacokinetic-pharmacodynamic considerations," Clinical Pharmacokinetics, vol. 30, no. 4, pp. 300-313, 1996.
[10] A. Fugh-Berman, "Herb-drug interactions," The Lancet, vol. 355, no. 9198, pp. 134-138, 2000.

[11] G. Valli and E.-G. V. Giardina, "Benefits, adverse effects and drug interactions of herbal therapies with cardiovascular effects," Journal of the American College of Cardiology, vol. 39, no. 7, pp. 1083-1095, 2002.

[12] A. Tachjian, V. Maria, and A. Jahangir, "Use of herbal products and potential interactions in patients with cardiovascular diseases," Journal of the American College of Cardiology, vol. 55, no. 6, pp. 515-525, 2010.

[13] Y. Huang, L.-J. Cui, Y.-H. Dou, and Y.-L. Wang, "Research on the interaction of mechanism between aspirin and human serum albumin," Chinese Pharmacological Bulletin, vol. 24, no. 9, pp. 1192-1195, 2008.

[14] É. A. Enyedy, E. Farkas, O. Dömötör, and M. A. Santos, "Interaction of folic acid and some matrix metalloproteinase (MMP) inhibitor folate- $\gamma$-hydroxamate derivatives with $\mathrm{Zn}$ (II) and human serum albumin," Journal of Inorganic Biochemistry, vol. 105, no. 3, pp. 444-453, 2011.

[15] J. Kang, Y. Liu, M.-X. Xie, S. Li, M. Jiang, and Y.-D. Wang, "Interactions of human serum albumin with chlorogenic acid and ferulic acid," Biochimica et Biophysica Acta, vol. 1674, no. 2, pp. 205-214, 2004.

[16] Y. Huang, L.-J. Cui, J.-M. Wang et al., "Comparative studies on interactions of baicalein, baicalin and scutellarin with lysozyme," European Journal of Medicinal Chemistry, vol. 46, no. 12, pp. 6039-6045, 2011.

[17] R. Liu, X. Y. Yang, and F. Y. Yang, "Monitoring of warfarin concentration in postoperative patients for preventing thrombosis," Northwest Pharmaceutical Journal, vol. 26, pp. 337-339, 2011.

[18] D. Yan, N. Zhao, X.-Y. Zhang, L. Yang, and B. Ma, "Pharmacokinetics and bioequivalence of sodium ferulate tablets in human," Chinese Journal of New Drugs, vol. 17, no. 3, pp. 247-250, 2008.

[19] Y. Huang, L.-J. Cui, J.-M. Wang et al., "Interaction of aconitine with bovine serum albumin and effect of atropine sulphate and glycyrrhizic acid on the binding," Journal of Luminescence, vol. 132, no. 2, pp. 357-361, 2012.

[20] L. J. Cui, J. M. Wang, and K. Huo, "Atropine sulfate against toxicity of mesaconitine by fluorescence spectra," Chinese Traditional and Herbal Drugs, vol. 43, pp. 1355-1360, 2012.

[21] N. Shahabadi and M. Maghsudi, "Binding studies of a new copper (II) complex containing mixed aliphatic and aromatic dinitrogen ligands with bovine serum albumin using different instrumental methods," Journal of Molecular Structure, vol. 929, no. 1-3, pp. 193-199, 2009.

[22] T. Yuan, A. M. Weljie, and H. J. Vogel, “Tryptophan fluorescence quenching by methionine and selenomethionine residues of calmodulin: orientation of peptide and protein binding," Biochemistry, vol. 37, no. 9, pp. 3187-3195, 1998.

[23] J. N. Miller, "Recent advances in molecular luminescence analysis," Proceedings of the Analytical Division of the Chemical Society, vol. 16, pp. 203-208, 1979.

[24] B. Klajnert and M. Bryszewska, "Fluorescence studies on PAMAM dendrimers interactions with bovine serum albumin," Bioelectrochemistry, vol. 55, no. 1-2, pp. 33-35, 2002.

[25] P. D. Ross and S. Subramanian, "Thermodynamics of protein association reactions: forces contributing to stability," Biochemistry, vol. 20, no. 11, pp. 3096-3102, 1981.

[26] P. O. Koh, "Ferulic acid attenuates the injury-induced decrease of protein phosphatase $2 \mathrm{~A}$ subunit B in ischemic brain injury," PLoS ONE, vol. 8, no. 1, Article ID e54217, 2013. 

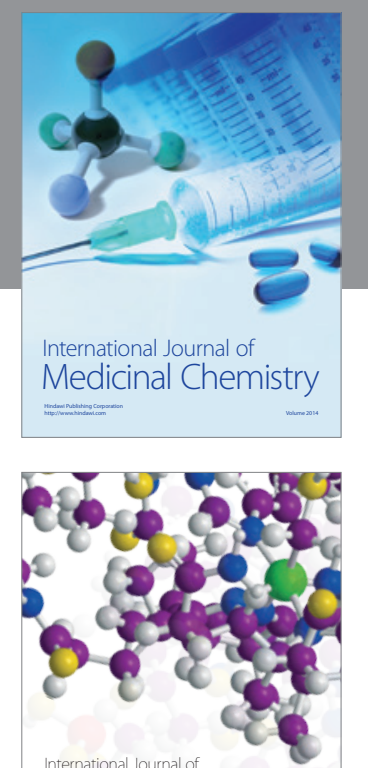

\section{Carbohydrate} Chemistry

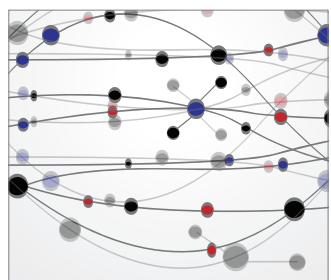

The Scientific World Journal
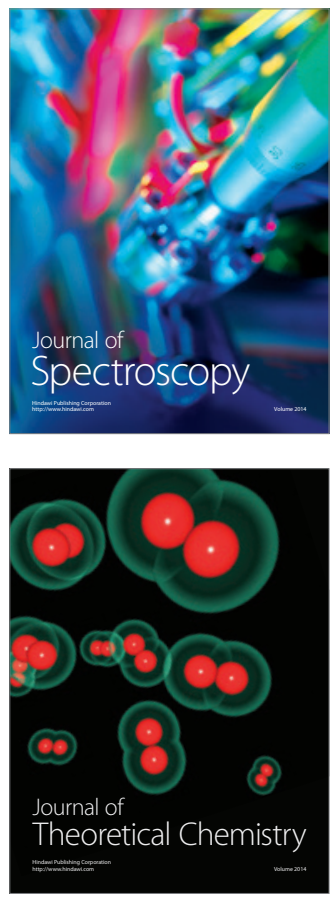
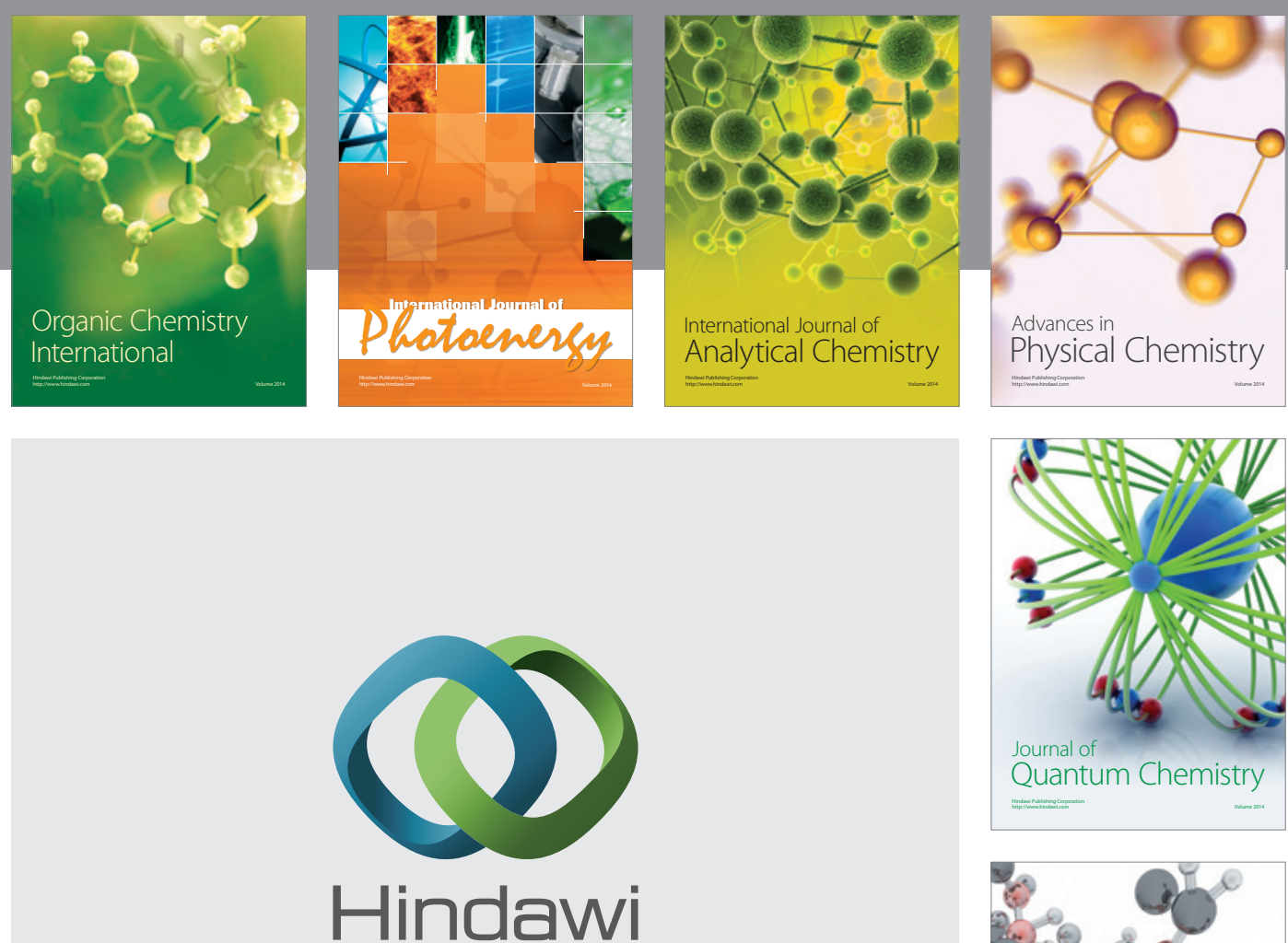

Submit your manuscripts at

http://www.hindawi.com

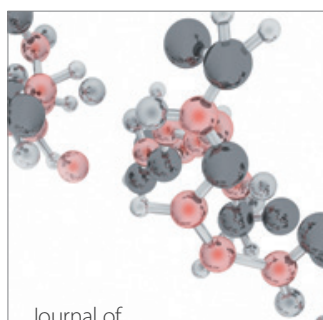

Analytical Methods

in Chemistry

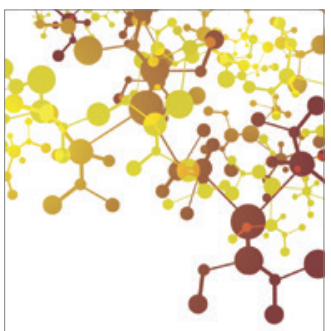

Journal of

Applied Chemistry

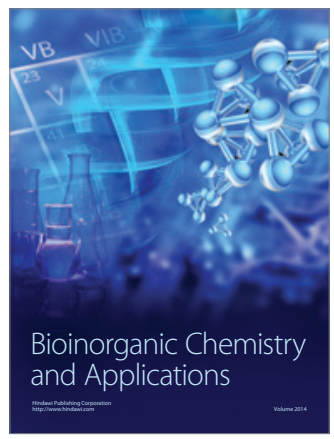

Inorganic Chemistry
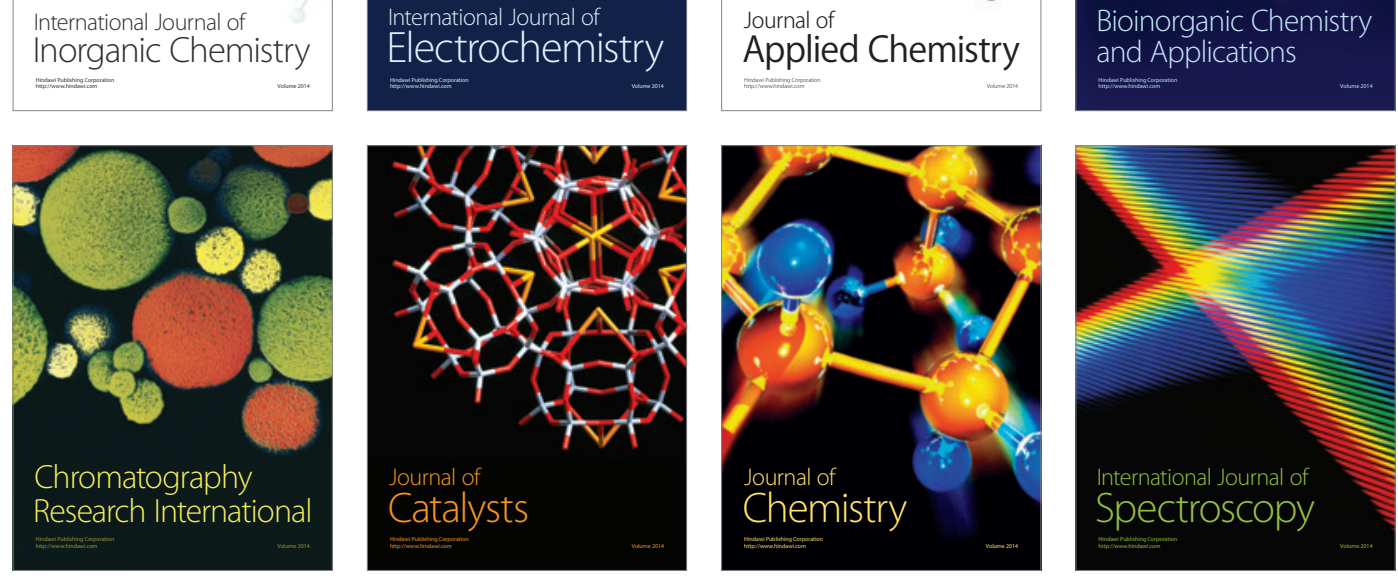